\title{
THE ROLE OF THE APPELL TRANSFORMATION IN THE THEORY OF HEAT CONDUCTION $\left.{ }^{1}\right)$
}

BY

D. V. WIDDER

1. Introduction. If $v(x, t)$ is an arbitrary function of two variables its Appell transform is

$$
\begin{aligned}
A[v] & =A_{x, t}[v]=k(x, t) v\left(\frac{x}{t}, \frac{-1}{t}\right), \\
k(x, t) & =\frac{e^{-x^{2} / 4 t}}{(4 \pi t)^{1 / 2}} .
\end{aligned}
$$

Here $k(x, t)$ is the fundamental solution of the heat equation

$$
\frac{\partial^{2} u}{\partial x^{2}}=\frac{\partial u}{\partial t} .
$$

As we shall see, this transformation serves in a remarkable way to establish a duality between types of solutions of (1.3). It was Appell [1] himself who showed that if $v$ is a solution then $A[v]$ is also. We shall be studying here various solutions which have integral representations and the effect of the Appell transformation thereon. A few of our results were outlined, mostly without proof, in [8].

Let us introduce notations for the various integral transforms to be considered, as follows:

Poisson transform

$$
P[\phi]=P_{x, t}[\phi]=\int_{-\infty}^{\infty} k(x-y, t) \phi(y) d y ;
$$

Fourier transform

$$
F[\phi]=F_{x, t}[\phi]=\int_{-\infty}^{\infty} e^{i x y-t y^{2}} \phi(y) d y ;
$$

Laplace transform

$$
L[\phi]=L_{x, t}[\phi]=\int_{-\infty}^{\infty} e^{x y+t v^{2}} \phi(y) d y .
$$

Received by the editors August 2, 1962 and, in revised form, October 28, 1962.

(1) This research was supported by the Air Force Office of Scientific Research, under Contract AF-AFOSR-62-198. 
All of these have kernels which are solutions of (1.3) for each value of the parameter $y$ and consequently produce solutions for more or less arbitrary functions $\phi$. The transform (1.4) is also commonly referred to as the Weierstrass or Gauss transform. It is clear that $P_{i x,-t}[\phi]$ is also a solution of (1.3). We shall see that this one is paired with $F[\phi]$ while $L[\phi]$ is paired with $P[\phi]$ in the duality mentioned above.

We list a number of examples which will be useful in the sequel.

A.

$$
P\left[x^{n}\right]=v_{n}(x, t)=n ! \sum_{k=0}^{[n / 2]} \frac{x^{n-2 k}}{(n-2 k) !} \frac{t^{k}}{k !}, \quad t>0 .
$$

This is called the heat polynomial of degree $n$. The Appell transform of this polynomial is the associated function studied in [6]:

$$
\begin{aligned}
A\left[v_{n}\right] & =w_{n}(x, t)=k(x, t) v_{n}(x / t,-1 / t) t^{-n}, & t>0 . \\
\text { B. } \quad F\left[e^{a x^{2}}\right] & =2 \pi k(x, t-a), & t>a . \\
\text { C. } \quad L\left[e^{-a x^{2}}\right] & =2 \pi k(i x, a-t), & t<a . \\
\text { D. } \quad A[k(x, t+a)] & =(4 \pi a)^{-1 / 2} k\left(x, t-a^{-1}\right), & t>a^{-1} .
\end{aligned}
$$

The transformed function is in every case a solution of (1.3) in the half-plane indicated.

We now state briefly a few of the results of the present paper. The Huygens property is defined in $\S 2$ below and the growth of an entire function, in $\S 4$. A fundamental result is the following.

$A$ function $u(x, t)$ is equal to the integral $F[\phi]$ defined by (1.5) where $\phi$ is entire of growth $(2,1 / \sigma)$, if and only if it is the Appell transform $A[v]$ of a temperature function $v(x, t)$ which has the Huygens property for $|t|<\sigma$.

We also obtain several new characterizations of positive temperature functions. The first involves those which are positive for all negative time.

$A$ solution $v(x, t)$ of $(1.3)$ is $\geqq 0$ for $t<0$ if and only if

$$
v(x, t)=\int_{-\infty}^{\infty} e^{x y+t y^{2}} d \alpha(y), \quad t<0,
$$

where $\alpha(y) \in \uparrow$.

An example of such a solution is $e^{t} \cosh x$, for which $\alpha$ is a step-function with jumps at \pm 1 .

We also characterize a subclass of the above functions as follows.

$A$ solution $v(x, t)$ of $(1.3)$ is $\geqq 0$ for $t<0$ and satisfies the inequality

$$
\int_{-\infty}^{\infty} v(x, t) e^{x^{2} / 4 t} d x<\infty, \quad t<0
$$

if and only if 


$$
v(x, t)=\int_{-\infty}^{\infty} k(y+i x,-t) \phi(y) d y, \quad t<0,
$$

where $\phi$ is positive definite.

A case in point is $v(x, t) \equiv 1$ with $\phi(y) \equiv 1$. A less trivial example is $v=k(i x, 1-t)$, which evidently satisfies the conditions of the theorem. Here $\phi$ is the positive definite function $e^{-y^{2} / 4} / \sqrt{ }(4 \pi)$.

Finally we characterize those temperature functions which are positive for positive time and are absolutely integrable in the space variable.

$A$ solution $u(x, t)$ of $(1.3)$ is $\geqq 0$ for $t<0$ and satisfies the inequality

$$
\int_{-\infty}^{\infty} u(x, t) d x<\infty, \quad t>0
$$

if and only if

$$
u(x, t)=\int_{-\infty}^{\infty} e^{i x y-\cdot y^{2}} \phi(y) d y, \quad t>0,
$$

where $\phi(y)$ is a positive definite function.

An example here is the fundamental solution itself with $\phi$ equal to the constant $(2 \pi)^{-1}$.

2. Relation between the Poisson and Fourier transforms. Let us reintroduce here some of the notation of [6].

Definition 2.1. A function $u(x, t) \in H$, or is a temperature function, for $a<t<b$ if and only if it is a class $C^{2}$ solution of the heat equation (1.3) there.

Definition 2.2. A function $u(x, t) \in H^{*}$, or has the Huygens property, for $a<t<b$ if and only if $u \in H$ there and

$$
u(x, t)=\int_{-\infty}^{\infty} k\left(x-y, t-t^{\prime}\right) u\left(y, t^{\prime}\right) d y
$$

for every $t, t^{\prime}, a<t^{\prime}<t<b$, the integral converging absolutely.

EXAMPLe A. Any function

$$
u(x, t)=\int_{-\infty}^{\infty} k(x-y, t) d \alpha(y)
$$

for which the integral converges absolutely in $a<t<b$ belongs to $H^{*}$ there. Equation (2.1) then results immediately by use of Fubini's theorem. In particular, a positive function of $H$ is also a function of $H^{*}$. See [7].

EXAmple B. The function $k(x, t+i)$ belongs to $H$ in the whole $x, t$-plane and belongs to $H^{*}$ in any strip of the form $-a<t<1 / a$. See [6 p. 242]. It is to be emphasized that a function may belong to $H^{*}$ on each of two overlapping sets without doing so on the sum of the sets. Thus the present function has the Huygens property for $-2<t<1 / 2$ and for $0<t<\infty$ but certainly not for 
$-2<t<2$, for example. If we chose $t^{\prime}=-1, t=1, x=0$ in the integral $(2.1$ it becomes

$$
\int_{-\infty}^{\infty} k(y, t) k(y,-1+i) d y .
$$

It clearly does not converge absolutely.

We prove now that any Poisson transform of a function in class $L$ is also a Fourier transform.

TheOREM 2. If $\phi \in L$ on $(-\infty, \infty)$, then $P_{x, t}[\phi] \in H^{*}$ for $t>0$ and

$$
P_{x, t}[\phi]=F_{x}{ }_{t}[\psi], \quad t>0,
$$

where

By definition

$$
\psi(x)=\frac{1}{2 \pi} \pi \int_{-\infty}^{\infty} e^{-i x y} \phi(y) d y
$$

$$
P_{x t}[\phi]=\int_{-\infty}^{\infty} k(x-y, t) \phi(y) d y, \quad t>0 .
$$

The integral converges absolutely for $t>0$ since $k \leqq(4 \pi t)^{-1 / 2}$. It consequently belongs to $H^{*}$ there by Example A of this section. By Example B of $\$ 1$ the integral (2.2) becomes

$$
\begin{aligned}
P_{x, t}[\phi] & =\frac{1}{2 \pi} \int_{-\infty}^{\infty} \phi(y) d y \int_{-\infty}^{\infty} e^{i(x-y) z-t z^{2}} d z \\
& =\frac{1}{2 \pi} \int_{-\infty}^{\infty} e^{i x z-t z^{2}} d z \int_{-\infty}^{\infty} e^{-i y z} \phi(y) d y \\
& =F_{x_{t}}[\psi] .
\end{aligned}
$$

The interchange in the order of integration is justified in an obvious way by Fubini's theorem.

3. Relation between the Poisson and Laplace transforms. To prove our next result we need a lemma.

Lemma 3. If $u(x, t) \in H^{*}$ for $a<t<b$, then $u(i x,-t) \in H^{*}$ for $-b<t<-a$.

Since the integral (2.1), when it converges, defines an entire function of $x$, the definition of $u(i x,-t)$ is clear. We must prove that if $a<t<t^{\prime}<b$ then

$$
u(i x, t)=\int_{-\infty}^{\infty} k\left(x-y, t^{\prime}-t\right) u\left(i y, t^{\prime}\right) d y,
$$

the integral converging absolutely. Choose $t^{\prime \prime}$ so that $a<t^{\prime \prime}<t$. Then by hypothesis 


$$
u(x, t)=\int_{-\infty}^{\infty} k\left(x-z, t-t^{\prime \prime}\right) u\left(z, t^{\prime \prime}\right) d z,
$$

the integral converging absolutely. Substituting (3.2) in the integral (3.1) we have

$$
\begin{aligned}
\int_{-\infty}^{\infty} k\left(x-y, t^{\prime}-t\right) d y \int_{-\infty}^{\infty} k\left(i y-z, t^{\prime}-t^{\prime \prime}\right) u\left(z, t^{\prime \prime}\right) d z \\
=\int_{-\infty}^{\infty} u\left(z, t^{\prime \prime}\right) d z \int_{-\infty}^{\infty} k\left(x-y, t^{\prime}-t\right) k\left(i y-z, t^{\prime}-t^{\prime \prime}\right) d y
\end{aligned}
$$

This last interchange is valid by virtue of the inequalities

$$
\begin{gathered}
\int_{-\infty}^{\infty} k\left(x-y, t^{\prime}-t\right) e^{y^{2} / 4\left(t^{\prime}-t^{\prime \prime}\right)} d y<\infty, \\
\int_{-\infty}^{\infty} e^{z^{2} / 4\left(t^{\prime}-t^{\prime \prime}\right)} u\left(z, t^{\prime \prime}\right) \mid d z<\infty .
\end{gathered}
$$

The integral (3.4) clearly converges since $t^{\prime \prime}<t$; (3.5) does also since (3.2) converges absolutely when $t=t^{\prime}$. But the value of the inner integral in (3.3) is known to be $k\left(i x-z, t-t^{\prime \prime}\right)$. See [5, p. 177]. That is, the integral (3.2) becomes

$$
\int_{-\infty}^{\infty} k\left(i x-z, t-t^{\prime \prime}\right) u\left(z, t^{\prime \prime}\right) d z
$$

and this by (3.2) is $u(i x, t)$. Thus (3.1) is proved. Finally, the absolute convergence of (3.1) follows as a consequence of Fubini's theorem.

THEOREM 3. If $\phi \in L$ on $(-\infty, \infty)$, then $P_{-i x-{ }_{-t}}[\phi] \in H^{*}$ for $t<0$ and

$$
P_{-i x,-t}[\phi]=L_{x, t}[\psi], \quad t<0,
$$

where

$$
\psi(x)=\frac{1}{2 \pi} \int_{-\infty}^{\infty} e^{-i x y} \phi(y) d y
$$

By definition

$$
P_{-i x,-t}[\phi]=\int_{-\infty}^{\infty} k(i x+y,-t) \phi(y) d y
$$

Since

$$
|k(i x+y,-t)| \leqq e^{-x^{2} / 4 t}(-4 \pi t)^{-1 / 2}, \quad t<0,
$$

it is clear that the integral (3.6) converges absolutely for $t<0$. Since the integral $P_{x, t}[\phi]$ converges absolutely for $t>0$ it defines a function of $H^{*}$ there. By Lemma 3 the function (3.6) belongs to $H^{*}$ for $t<0$. By Example C of $\S 1(a=0)$ the integral (3.6) becomes 


$$
\frac{1}{2 \pi} \int_{-\infty}^{\infty} \phi(y) d y \int_{-\infty}^{\infty} e^{z(x-i y)+t z^{2}} d z=\frac{1}{2 \pi} \int_{-\infty}^{\infty} e^{z x+t z^{2}} d z \int_{-\infty}^{\infty} e^{-i y z} \phi(y) d y
$$

The interchange of integrals is obviously permissible for $t<0$, and the inner integral is $\psi(z)$ as predicted.

Note that the initial temperature of $F_{x, t}[\psi]$ is the Fourier transform of $\psi$; the terminal temperature of $L_{x, t}[\psi]$ is the Laplace transform of $\psi$ when these transforms exist.

4. A criterion for the Fourier representation. Let us recall the following definition. DeFINITION 4. An entire function

$$
f(x)=\sum_{n=0}^{\infty} a_{n} x^{n}
$$

belongs to the class $(2, \sigma)$, or has growth $(2, \sigma)$, if and only if

$$
\lim _{n \rightarrow \infty} \sup n\left|a_{n}\right|^{2 / n} \leqq 2 e \sigma .
$$

See, for example, [2, p. 8]. A function of this class is of order $\leqq 2$, and if it is of order 2 , then it is of type $\leqq \sigma$. For example, the functions $x e^{-3 x^{2}}, e^{x^{2}}, e^{5 x}$ all belong to $(2,3)$, whereas $e^{-x^{4}}$ does not.

We first call attention to the following lemma.

LEMMA 4.1. If $s>0, t>0$, then

$$
A_{x, t}[k(x-y, t+s)]=A_{y, s}[k(x-y, t+s)] .
$$

This is a simple identity. Both sides are equal to

$$
(4 \pi)^{-1}(s t-1)^{-1 / 2} \exp \left[\left(2 x y-t y^{2}-s x^{2}\right)(4 s t-4)^{-1}\right] .
$$

A second preliminary result is contained in

Lemma 4.2. If $v(x, t) \in H^{*}$ for $|t|<\sigma$, then $v(-2 i x, 0) \in(2,1 / \sigma)$.

To prove this we appeal to Theorem 11.1 of [6] and thus admit that

$$
\begin{array}{ll}
v(x, t)=\sum_{n=0}^{\infty} a_{n} v_{n}(x, t), & |t|<0 \\
v(x, 0)=\sum_{n=0}^{\infty} a_{n} x^{n} . &
\end{array}
$$

By Theorem 5.5 of $[6], v(x, 0) \in(2,1 / 4 \sigma)$. Thus (4.1) shows that $v(-2 i x, 0)$ $\in(2,1 / \sigma)$, as stated.

THEOREM 4. A necessary and sufficient condition that

$$
u(x, t)=F[\phi]=\int_{-\infty}^{\infty} e^{x y-t y^{2}} \phi(y) d y, \quad t>1 / \sigma,
$$


where $\phi(y) \in\left(2, \sigma^{-1}\right)$, is that there should exist a function $v(x, t)$ of $H^{*}$ in $|t|<\sigma$ such that $u=A[v]$.

We prove first the sufficiency. Since $v \in H^{*}$ for $|t|<\sigma$, then for any positive $\sigma^{\prime}<\sigma$

$$
v(x, t)=\int_{-\infty}^{\infty} k\left(x-y, t+\sigma^{\prime}\right) v\left(y,-\sigma^{\prime}\right) d y,
$$

the integral converging absolutely when $-\sigma^{\prime}<t<\sigma$. By Lemma 4.1

$$
\begin{aligned}
A[v] & =\int_{-\infty}^{\infty} k\left(y, \sigma^{\prime}\right) k\left(x-\frac{y}{\sigma^{\prime}}, t-\frac{1}{\sigma^{\prime}}\right) v\left(y,-\sigma^{\prime}\right) d y, \\
& =\frac{1}{2 \pi} \int_{-\infty}^{\infty} k\left(y, \sigma^{\prime}\right) v\left(y,-\sigma^{\prime}\right) d y \int_{-\infty}^{\infty} \exp \left[i z\left(x-\frac{y}{\sigma^{\prime}}\right)-z^{2}\left(t-\frac{1}{\sigma^{\prime}}\right)\right] d z .
\end{aligned}
$$

Here we have used Example B of $\S 1$. If interchange of iterated integrals is permitted, we obtain

$$
\begin{aligned}
A[v] & =\frac{1}{2 \pi} \int_{-\infty}^{\infty} \exp \left(i x z-t z^{2}\right) d z \int_{-\infty}^{\infty} v\left(y,-\sigma^{\prime}\right) k\left(-2 i z-y, \sigma^{\prime}\right) d y & \\
& =\int_{-\infty}^{\infty} e^{i x z-t z^{2}} \phi(z) d z, & t>1 / \sigma^{\prime},
\end{aligned}
$$

where $2 \pi \phi(z)=v(-2 i z, 0)$. To justify the interchange it is sufficient to know, that

$$
\int_{-\infty}^{\infty} \exp \left(-t z^{2}+\frac{z^{2}}{\sigma^{\prime}}\right) d z \int_{-\infty}^{\infty} \exp \left(-\frac{y^{2}}{4 \sigma^{\prime}}\right)\left|v\left(y,-\sigma^{\prime}\right)\right| d y<\infty
$$

The inner integral is finite since (4.2) converges absolutely when $x=t=0$. The outer integral clearly converges when $t>1 / \sigma^{\prime}$. Since $\phi(z)$ is independent of $\sigma^{\prime}$, it is clear that (4.3) continues to hold for all $t>1 / \sigma$. Finally, that $\phi \in(2,1 / \sigma)$ follows from Lemma 4.2 .

To prove the necessity we now assume that $u=F[\phi]$ with $\phi$ belonging to $(2,1 / \sigma)$. We appeal to Theorem 12.1 of [6] to show that

where

$$
u(x, t)=\sum_{n=0}^{\infty} b_{n} w_{n}(x, t), \quad t>1 / \sigma,
$$

$$
b_{n}=\frac{2 \pi \phi^{(n)}(v)}{n !(-2 i)^{n}}
$$

Since $w_{n}=A\left[v_{n}\right]$ it is clear that $u=A[v]$, where

$$
v(x, t)=\sum_{n=0}^{\infty} b_{n} v_{n}(x, t), \quad-\sigma<t<0 .
$$


By Theorem 11.1 of [6] the sum of any such series belongs to $H^{*}$ throughout its region of convergence, always an entire strip of the form $|t|<\sigma$. The convergence of (4.5) in $-\sigma<t<0$ implies its convergence in $|t|<\sigma$, so that $v \in H^{*}$ there. The equation (4.4) yields

$$
\begin{aligned}
v(x, 0) & =2 \pi \sum_{n=0}^{\infty} \frac{\phi^{(n)}(0)}{n !}\left(\frac{x}{-2 i}\right)^{n}=2 \pi \phi\left(\frac{i x}{2}\right) \\
v(-2 i z, 0) & =2 \pi \phi(z),
\end{aligned}
$$

thus confirming the relation between $v$ and $\phi$ established in the sufficiency proof.

Corollary 4. If $v(x, t) \in H^{*}$ for $a<t<b$ and if $a<t_{0}<b$, then

$$
F_{x, t}\left[v\left(-2 i x, t_{0}\right)\right]=2 \pi A_{x, t}\left[v\left(x, t+t_{0}\right), \quad t>1 / \sigma,\right.
$$

where $\sigma=\min \left(t_{0}-a b-t_{0}\right)$.

This result was stated as Theorem II in [8]. It follows here by applying Theorem 4 to $v\left(x, t+t_{0}\right)$, which belongs to $H^{*}$ in $|t|<\sigma$.

Example $D$ of $\S 1$ provides an illustration of Theorem 4. The function $v=k(x, t+a) \in H^{*}$ for $t>-a$ but the largest strip of the form $|t|<\sigma$ contained in that half-plane is $|t|<a$. Hence $A[v]$, according to Theorem 4, must equal $F[\phi]$ for $t>1 / a$, where

$$
\begin{aligned}
2 \pi \phi(y) & =v(-2 i y, 0)=e^{y^{2} / a}(4 \pi a)^{-1 / 2}, \\
A[\phi] & =\frac{(4 \pi a)^{-1 / 2}}{2 \pi} \int_{-\infty}^{\infty} e^{i x y-t y^{2}} e^{y^{2} / a} d y, \\
A[\phi] & =(4 \pi a)^{-1 / 2} k\left(x, t-\frac{1}{a}\right),
\end{aligned}
$$

Notice that $\phi \in(2,1 / a)$ as predicted and that (4.6) converges in no larger region than that predicted by the theorem.

5. A sufficient condition for the Fourier representation. Although the previous example shows that Theorem 4 cannot be improved to give a larger region of convergence for the integral (4.6), nevertheless for some examples the region is indeed larger. Consider the function

$$
v(x, t)=k(i x, \delta-t)+k(x, t+\sigma) .
$$

It belongs to $H^{*}$ in $-\sigma<t<\delta$. If $\sigma>\delta$, Theorem 4 would predict the convergence of the corresponding integral $A[\phi]$ for $t>1 / \delta$. However

$$
\phi(y)=\frac{e^{-y^{2} / \delta}}{4 \pi \sqrt{ } \delta}+\frac{e^{y^{2} / \sigma}}{4 \pi \sqrt{ } \sigma},
$$

so that $F[\phi]$ actually converges in the larger region $t>1 / \sigma$. The following theorem would cover such an example. 
THEOREM 5. If $v(x, t) \in H^{*}$ for $-\sigma<t<\delta, \sigma>0, \delta>0$, then

$$
A[v]=F[\phi],
$$

where $2 \pi \phi(y)=v(-2 i y, 0)$ is entire and

$$
\lim _{x \rightarrow \pm \infty} \sup ^{-2} \log |\phi(x)| \leqq 1 / \sigma .
$$

Note that if $\sigma>\delta$ then $\phi \in(2,1 / \delta)$ and Theorem 4 would guarantee the convergence of the integral $F[\phi]$ for $t>1 / \delta$, in contrast with the present $t>1 / \sigma$.

The proof of (5.1) proceeds exactly as in the sufficiency of the conditions of Theorem 4. We find as before that $\phi(y)=v(-2 i y, 0) / 2 \pi$. But now we cannot show that $\phi \in(2,1 / \sigma)$. We show instead that (5.2) holds. From equation (4.2) we have

$$
\mid v\left(-2 i x, 0\left|\leqq \frac{e^{x^{2} / \sigma^{\prime}}}{\sqrt{ } 4 \pi \sigma^{\prime}} \int_{-\infty}^{\infty} e^{-y^{2} / 4 \sigma^{\prime}}\right| v\left(y,-\sigma^{\prime}\right) \mid d y,\right.
$$

from which it follows that

$$
\lim _{x \rightarrow \pm \infty} \sup ^{-2} \log |\phi(x)| \leqq 1 / \sigma^{\prime}
$$

On account of the arbitrary nature of $\sigma^{\prime}$ this inequality implies (5.2), and the proof is complete.

Observe that the condition $\delta>0$ in Theorem 5 cannot be improved. The function $v=k(i x,-t) \in H^{*}$ in $-\infty<t<0$. Its Appell transform is the constant $1 /(4 \pi)$. This certainly has no Fourier representation $A[\phi]$, since any such function must vanish at infinity.

6. A necessary condition for the Fourier representation. It should be observed that $F[\phi]$ may define a temperature function even when $\phi \notin(2, \sigma)$ for any $\sigma$. For example, if $\phi(y)=e^{-y^{4}}, \phi$ is entire of order 4. But the integral

$$
F[\phi]=\int_{-\infty}^{\infty} e^{i x y-t y^{2}} e^{-y^{4}} d y
$$

converges over the whole $x, t$-plane. Although the example is not covered by Theorem 4, $F[\phi]$ is nevertheless the Appell transform of some function, which of course cannot belong to $H^{*}$ in any strip including the $x$-axis. The following result would include such an example.

THEOREM 6. If $u(x, t)=F[\phi]=\int_{-\infty}^{\infty} e^{i x y-t y^{2}} \phi(y) d y, t>1 / \sigma \geqq 0$, where

$$
\limsup _{x \rightarrow \pm \infty} x^{-2} \log |\phi(x)| \leqq 1 / \sigma,
$$

then there exists $v(x, t)$ belonging to $H^{*}$ in $-\sigma<t<0$ such that $A[v]=u$.

We define $v$ explicitly:

$$
v(x, t)=2 \pi \int_{-\infty}^{\infty} k(y+i x,-t) \phi(-y / 2) d y .
$$


By hypothesis

$$
\phi(y)=O\left(e^{y^{2} / \sigma^{\prime}}\right),
$$$$
y \rightarrow \pm \infty
$$

for any positive $\sigma^{\prime}<\sigma$. Thus the integral (6.2) is dominated by

$$
(-4 \pi t)^{-1 / 2} e^{-x^{2} / 4 t} \int_{-\infty}^{\infty} e^{y^{2} / 4 t} O\left(e^{y^{2} / 4 \sigma^{\prime}}\right) d y .
$$

As a consequence it converges for $-\sigma^{\prime}<t<0$ and hence also for $-\sigma<t<0$. The same relation (6.3) shows that the integral

$$
w(x, t)=2 \pi \int_{-\infty}^{\infty} k(x-y, t) \phi(-y / 2) d y
$$

converges absolutely for $0<t<\sigma$, and defines a function of $H^{*}$ there. Since $v(x, t)=w(-i x,-t)$, we see by Lemma 3 that $v(x, t) \in H^{*}$ for $-\sigma<t<0$. But from the definition of the Appell transformation (1.1) we have

$$
\begin{aligned}
A[v] & =\frac{1}{2} \int_{-\infty}^{\infty} e^{-i x y / 2-y^{2} t / 4} \phi(-y / 2) d y \\
& =\int_{-\infty}^{\infty} e^{i x y-t y^{2}} \phi(y) d y=F[\phi]=u .
\end{aligned}
$$

This completes the proof.

In the example of this section the left-hand side of (6.1) is $-\infty$ so that $\sigma$ may be taken $+\infty$. The function (6.2) belongs to $H^{*}$ for $-\infty<t<0$.

7. The Laplace transform of an Appell transform. If a function $v(x, t) \in H^{*}$ in a strip including the $x$-axis, then the Laplace transform of $A[v]$ has a remarkably simple form. It is in fact equal to $v(2 x, 0) e^{t x^{2}}$ for all $t$ sufficiently large. The explicit result follows.

THEOREM 7. If $v(x, t) \in H^{*}$ for $|t|<\sigma$, then for any $t>1 / \sigma$

$$
v(2 x, 0) e^{t x^{2}}=\int_{-\infty}^{\infty} e^{x y} A_{y . t}[v] d y .
$$

Make the change of variable $y=t z$ in the integral (7.1) multiplied by $e^{-t x^{2}}$. We obtain

$$
\begin{aligned}
t e^{-t x^{2}} \int_{-\infty}^{\infty} e^{x t z} k(t z, t) v(z,-1 / t) d z & =(t / 4 \pi)^{1 / 2} \int_{-\infty}^{\infty} e^{-(2 x-z)^{2} t / 4} v(z,-1 / t) d z \\
& =v(2 x, 0)
\end{aligned}
$$

This last equation follows from the Huygens property provided only that $-\sigma<-1 / t<0$, and this is guaranteed by hypothesis. This completes the proof.

This result as applied to a function $v\left(x, t+t_{0}\right)$ was stated without proof as Theorem III in [7]. As an example take $v=k(x, t+a)$. Then for $t>1 / a$ 


$$
e^{t x^{2}} e^{-x^{2} / a}=\int_{-\infty}^{\infty} e^{x y} k\left(y, t-\frac{1}{a}\right) d y .
$$

But this is a familiar result, which can be verified by Example $C$ of $\S 1$.

8. Positive temperatures in negative time. Temperature functions which are positive for all negative time, such as $e^{t} \cosh x$, have a particularly simple representation in terms of the Laplace integral. The result follows.

THEOREM 8.1. A necessary and sufficient condition that

$$
v(x, t)=\int_{-\infty}^{\infty} e^{x y+t y^{2}} d \alpha(y)
$$

where $\alpha(y) \in \uparrow$ and the integral converges for $-\infty<t<0$ is that $v(x, t) \geqq 0$ and $v(x, t) \in H$ for $-\infty<t<0$.

The necessity of the condition is trivial. The kernel of (8.1) belongs to $H$ for each $y$, and differentiation under the integral sign is valid for Laplace integrals.

Conversely, apply the Appell transform to the non-negative temperature function $v$ :

$$
A[v]=k(x, t) v\left(\frac{x}{t},-\frac{1}{t}\right) .
$$

Clearly $A[v] \in H$ and is $\geqq 0$ for $t>0$. Hence by an earlier result of the author [7] it has a Poisson representation

$$
\begin{aligned}
A[v] & =\int_{-\infty}^{\infty} k(x-y, t) d \beta(y), & 0<t<\infty \\
v\left(\frac{x}{t},-\frac{1}{t}\right) & =e^{x^{2} / 4 t} \int_{-\infty}^{\infty} e^{-(x-y)^{2} / 4 t} d \beta(y), &
\end{aligned}
$$

where $\beta(y) \in \uparrow$. Now by obvious change of variable

$$
v(x, t)=\int_{-\infty}^{\infty} e^{x y+t y^{2}} d \beta(y / 2), \quad-\infty<t<0 .
$$

The proof is now concluded by defining $\alpha(y)$ as $\beta(y / 2)$.

Corollary 8.1. A necessary and sufficient condition that

$$
v(x, t)=\int_{-\infty}^{\infty} e^{x y+t y^{2}} d \alpha(y)
$$

where $\alpha(y) \in \uparrow$ and the integral converges for $-\infty<t<c$, is that $v(x, t) \geqq 0$ and $v(x, t) \in H$ there.

This is proved by applying the theorem to $v(x, t+c)$. 
As an application of this theorem we prove an earlier result of I. I. Hirsch$\operatorname{man}[4]$.

THEOREM 8.2. If $v(x, t) \in H$ and is $\geqq 0$ for $-\infty<t \leqq c$ and if $\operatorname{Max}_{|x| \leqq r} v(x, t)=M(r)$, then $\liminf _{r \rightarrow \infty} \log M(r) \leqq 0$ implies that $u(x, t)$ is constant for $-\infty<t \leqq c$.

We may assume that $c=0$ and show that the function $\alpha(y)$ of (8.1) has at most one point of increase, at $y=0$. Suppose on the contrary that $y=y_{0} \neq 0$ is such a point. Choose $\delta$ so that $y=0$ is not in the interval $\left(y_{0}-\delta, y_{0}+\delta\right)$. Then

$$
v(x, 0) \geqq \int_{y_{0}-\delta}^{y_{0}+\delta} e^{x y} d \alpha(y)>p e^{\left(y_{0}-\delta\right) x}\left[>p e^{\left(y_{0}+\delta\right) x}\right], \quad x>0[x<0]
$$

where $p$ is the positive number $\alpha\left(y_{0}+\delta\right)-\alpha\left(y_{0}-\delta\right)$. This shows that

In either case

$$
\begin{array}{rlrl}
M(r) & \geqq p e^{\left(y_{0}-\delta\right) r}, & y_{0}-\delta>0 \\
& \geqq p e^{-\left(y_{0}+\delta\right) r}, & & y_{0}+\delta<0 .
\end{array}
$$

$$
\liminf _{r \rightarrow \infty} r^{-1} \log M(r)>0,
$$

contradicting the hypothesis. This concludes the proof.

As a consequence of this theorem it is clear that any temperature function which is uniformly bounded for $-\infty<t \leqq c$ is a constant.

9. A subclass of positive temperatures in negative time. If an additional condition is imposed on the function $v(x, t)$ of Theorem 8.1 it will have in addition to (8.1) a quite different integral representation.

THEOREM 9. A necessary and sufficient condition that

$$
v(x, t)=\int_{-\infty}^{\infty} k(y+i x,-t) \phi(y) d y, \quad-\infty<t<0,
$$

where $\phi(y)$ is positive definite, is that for $-\infty<t<0 \quad v(x, t) \in H, \geqq 0$ and that for some $t_{0}<0$

$$
\int_{-\infty}^{\infty} v\left(x, t_{0}\right) e^{x^{2} / 4 t_{0}} d x<\infty
$$

We prove first the necessity. Assume the representation (9.1). Since $\phi$ is positive definite we have by Bochner's theorem [3, p. 76], that for some $\alpha(z)$ that is nondecreasing and bounded

$$
\phi(y)=\int_{-\infty}^{\infty} e^{i y z} d \alpha(z) .
$$

Hence 


$$
v(x, t)=\int_{-\infty}^{\infty} k(y+i x,-t) d y \int_{-\infty}^{\infty} e^{i y z} d \alpha(z)=\int_{-\infty}^{\infty} d x(z) \int_{-\infty}^{\infty} k(y+i x,-t) e^{i y z} d y .
$$

The interchange is valid for $t<0$ since

$$
\int_{-\infty}^{\infty} d \alpha(z) \int_{-\infty}^{\infty} e^{y^{2} / 4 t} d y<\infty
$$

Evaluating the inner integral (9.4) we obtain

$$
v(x, t)=\int_{-\infty}^{\infty} e^{x z+t z^{2}} d \alpha(z)
$$

from which it is clear that $v \in H, \geqq 0$. Moreover,

$$
\begin{array}{rlrl}
\int_{-\infty}^{\infty} v(x, t) e^{x^{2} / 4 t} d x & =\int_{-\infty}^{\infty} e^{t z^{2}} d \alpha(z) \int_{-\infty}^{\infty} e^{x z} e^{x^{2} / 4 t} d x \\
& =\sqrt{ }(4 \pi(-t)) \int_{-\infty}^{\infty} d \alpha(z)<\infty, & & t<0
\end{array}
$$

Hence (9.2) is also established.

Conversely, if $v(x, t) \in H$, $\geqq 0$, for $-\infty<t<0$, then by Theorem 8.1 equation (9.5) holds for some nondecreasing function $\alpha(z)$. Since (9.2) is now assumed, it is the left side of (9.6) that is known to be finite for some $t_{0}$. From the right side we then conclude that $\alpha(z)$ is also bounded. Hence we may define $\phi(y)$ by (9.3) as a positive definite function. Finally, the equality of the integrals (9.5) and (9.1), already established, concludes the proof.

To show that the functions here considered really form a proper subclass of those considered in $\S 8$ we have only to exhibit the function $v=k(i x,-t)$. It fails to satisfy (9.2) for any $t_{0}$. Yet it is positive for $t<0$ and so has the representation (8.1). In fact $\alpha(y)=y /(2 \pi)$.

10. Positive temperatures in positive time. As we have noted above, temperature functions which are positive for $t>0$ have a Poisson-Stieltjes integral representation. However, there is a subset of these functions which also have the Fourier integral representation.

THEOREM 10. A necessary and sufficient condition that

$$
u(x, t)=F[\phi]=\int_{-\infty}^{\infty} e^{i x y-t y^{2}} \phi(y) d y, \quad 0<t<\infty,
$$

where $\phi(y)$ is positive definite, is that $u(x, t) \in H, \geqq 0$ for $0<t<\infty$ and $\in L(-\infty<x<\infty)$ for some $t=t_{0}>0$.

We prove first the sufficiency. Assuming $u \in H, \geqq 0$, a new appeal to [7] gives

$$
u=\int_{-\infty}^{\infty} k(x-y, t) d \alpha(y), \quad 0<t<\infty
$$

for some $\alpha(y) \in \uparrow$. Since $u\left(x, t_{0}\right) \in L$ we have 


$$
\begin{aligned}
\int_{-\infty}^{\infty} d x \int_{-\infty}^{\infty} k\left(x-y, t_{0}\right) d x(y) & =\int_{-\infty}^{\infty} d \alpha(y) \int_{-\infty}^{\infty} k\left(x-y, t_{0}\right) d x \\
& =\int_{-\infty}^{\infty} d \alpha(y)<\infty
\end{aligned}
$$

Hence $\alpha(y)$ is bounded and we can form the positive definite function

$$
\phi(y)=\frac{1}{2 \pi} \int_{-\infty}^{\infty} e^{-i y z} d \alpha(z) .
$$

Substitute this integral for $\phi(y)$ in (10.1) to obtain

$$
\begin{aligned}
\frac{1}{2 \pi} \int_{-\infty}^{\infty} e^{i x y-t y^{2}} d y \int_{-\infty}^{\infty} e^{-i y z} d \alpha(z) & =\frac{1}{2 \pi} \int_{-\infty}^{\infty} d \alpha(z) \int_{-\infty}^{\infty} e^{i(x-z) y-t y^{2}} d y \\
& =\int_{-\infty}^{\infty} k(x-z, t) d \alpha(z)=u(x, t), \quad 0<t<\infty .
\end{aligned}
$$

The interchange of integrals is valid for $t>0$ since $\alpha(z)$ is bounded.

Conversely, if we assume the representation (10.1) then $\phi(y)$ is given by (10.4) according to Bochner's theorem. And now $\alpha(z)$ is known to be bounded and equation (10.5) results as before. It shows that $u \in H, \geqq 0$, for $t>0$. Finally $u\left(x, t_{0}\right) \in L$ for every $t_{0}>0$ from (10.2) and (10.3). This completes the proof.

A simple example of a positive temperature function which does not belong to $L$ on any line $t=t_{0}$ is $u=x^{2}+2 t$. It has the representation (10.5) with $\alpha(z)=z^{3} / 3$, but of course none of the form (10.1).

An example of the theorem is $k(x, t+a), a>0$. Here $\phi(x)=e^{-a x^{2}} /(2 \pi)$ and $k \in L$ for each $t>0$.

\section{BIBLIOGRAPHY}

1. P. Appell, Sur l'équation $\partial^{2} z / \partial x^{2}-\partial z / \partial y$ et la théorie de la chaleur, J. Math. Pures Appl. 8 (1892), 187-216.

2. R. P. Boas, Entire functions, Academic Press, New York, 1954.

3. S. Bochner, Vorlesungen über Fouriersche Integrale, 1932.

4. I. I. Hirschman, A note on the heat equation, Duke Math. J. 19 (1952), 487-492.

5. I. I. Hirschman and D. V. Widder, The convolution transform, Princeton Univ. Press, Princeton, N. J., 1955.

6. P. C. Rosenbloom and D. V. Widder, Expansions in terms of heat polynomials and associated functions, Trans. Amer. Math. Soc. 92 (1959), 220-266.

7. D. V. Widder, Positive temperatures on an infinite rod, Trans. Amer. Math. Soc. 55 (1944), 85-95.

8. - Transformations associées à l'équation de la chaleur, C. R. Acad. Sci. Paris, 253 (1961), 915-917.

HARVARD UNIVERSITY,

CAMBridge, MASSACHUSETTS 